\title{
Characterization of Mycobacterium tuberculosis ribosome recycling factor (RRF) and a mutant lacking six amino acids from the C-terminal end reveals that the C-terminal residues are important for its occupancy on the ribosome
}

\author{
Arasada Rajeswara Rao and Umesh Varshney
}

Author for correspondence: Umesh Varshney. Tel: +91 80394 2686. Fax: +91 803602697 or 0683. e-mail: varshney@mcbl.iisc.ernet.in

Department of Microbiology and Cell Biology, Indian Institute of Science, Bangalore, 560012, India

\begin{abstract}
Ribosome recycling factor (RRF), coded for by the frr locus, is involved in the disassembly of post-termination complexes and recycling of the ribosomes for a fresh round of initiation in bacteria and in eukaryotic organelles. In a crossspecies-complementation experiment, it was shown that the Thermus thermophilus RRF protein lacking five amino acids from its C-terminal end ( $\triangle$ C5TthRRF) but not the full-length protein (TthRRF) complemented Escherichia coli for its frrts phenotype. It was also shown that the Mycobacterium tuberculosis RFF protein (MtuRRF) did not complement E. coli LJ14 for frrts. However, simultaneous expression of elongation factor G (EFG) and RRF from $M$. tuberculosis resulted in complementation of $E$. coli LJ14. Here it is shown that unlike $\triangle$ C5TthRRF, an equivalent mutant of MtuRRF lacking six amino acids from its C-terminal end ( $\triangle$ C6MtuRRF) did not complement $E$. coli LJ14. Surprisingly, $\triangle$ C6MtuRRF failed to complement the strain even in the presence of homologous EFG (MtuEFG). The biochemical and biophysical characterization of these proteins suggested that the mutant RRF folded properly. However, ribosome-binding assays showed that the mutant protein was compromised in its binding to $E$. coli ribosomes. It is suggested that the conserved amino acids at the C-terminal end of the RRFs contribute to their residency on ribosomes and that the specific interactions between RRF and EFG are crucial in the disassembly of the termination complex.
\end{abstract}

Keywords: tubercle bacilli, Thermus thermophilus, elongation factor G, polysome binding, termination complex

\section{INTRODUCTION}

Mycobacterium tuberculosis is responsible for more casualties worldwide than any other infectious agent. The reemergence of tuberculosis as a serious infection, especially in AIDS patients, and the availability of the complete genome sequence of $M$. tuberculosis have been

\footnotetext{
Abbreviations: $\mathrm{CD}$, circular dichroism; EcoEFG, E. coli EFG; EcoRRF, fulllength E. coli RRF; EFG, elongation factor G; frr, locus coding for RRF; frrts, locus coding for temperature-sensitive RRF; ESI-MS, electron spray ionization-mass spectroscopy; MtuEFG, $M$. tuberculosis EFG; MtuRRF, fulllength $M$. tuberculosis RRF; RFF, ribosome recycling factor; TthRRF, fulllength $T$. thermophilus RRF.
}

the major driving forces behind much of the recent research on this organism (Clark-Curtis, 1990; Young \& Cole, 1993; Kaufmann \& van Embden, 1993; McFadden, 1996; Cole et al., 1998). The structural and functional analyses of the essential gene products associated with tubercle bacilli constitute a crucial component in the discovery of new drug targets to control the growth of M. tuberculosis.

Ribosome recycling factor (RRF) is an essential factor for protein synthesis in bacteria and in eukaryotic organelles (Janosi et al., 1996; Kaji et al., 1998). RRF is required for the disassembly of the post-termination complex. Interestingly, in eukaryotes RRF is only needed 


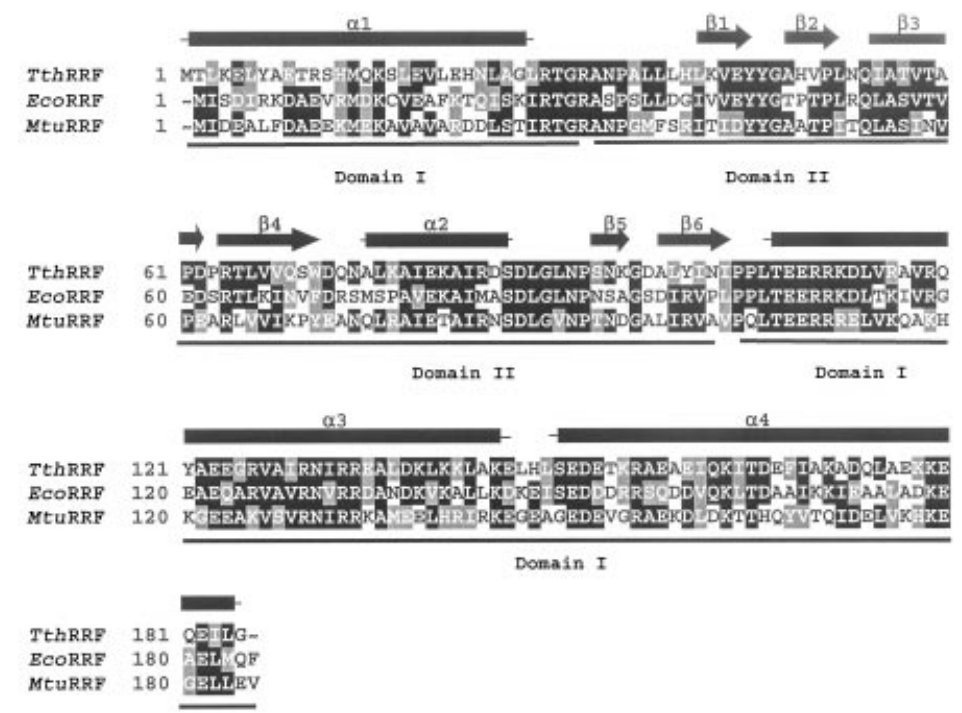

Fig. 1. Sequence alignment of MtuRRF, ECORRF and TthRRF. Conserved residues are highlighted white on a black background; similar residues are shown white on a grey background. Various structural elements in domains I and II (as in EcoRRF) are depicted. The $\alpha$-helices and $\beta$ strands are shown as solid boxes and arrows, respectively. In EcoRRF, domain I consists of residues 1-30 and 104-185, whereas the intervening residues (32-102) constitute domain II (Kim et al., 2000). The sequence alignment was done using PILEUP and CLUSTAL W. BOXSHADE was used to obtain the colour-shaded schematic representation.

in the organelles, making it a novel drug target (Janosi et al., 1996; Kaji et al., 1998). Also, in Staphylococcus aureus the levels of RRF increase upon infection of animal cells (Lowe et al., 1998). Furthermore, in brucellosis (caused by Brucella melitensis) the sera from infected sheep show antibodies against RRF, suggesting this protein to be a virulence factor (Vizcaino et al., 1996). Thus, RRF may even be of interest in developing subcellular vaccines. The RRFs from different bacteria are highly conserved in their primary structure (Fig. 1), and the three-dimensional structure of the RRFs from four organisms (Thermotoga maritima, Thermus thermophilus, Escherichia coli and Aquifex aeolicus) are known (Selmer et al., 1999; Toyada et al., 2000; Kim et al., 2000; Yoshida et al., 2001). These structures show that the overall architecture of the different RRFs, consisting of two domains, is also highly conserved and mimics tRNAs in its size and shape. Domain I is represented by three long $\alpha$-helices and domain II is composed of a $\beta-\alpha-\beta$ sandwich. The two domains are connected to each other by two loops. Domains I and II represent the long and short arms, respectively, of the ' $L$ '-shaped tRNA.

In spite of the remarkable structural similarity between the different RRFs, cross-genus-complementation experiments show that while the RRF from Pseudomonas aeruginosa functions efficiently in $E$. coli, the RRF from T. thermophilus (TthRRF) does not (Ohnishi et al., 1999; Fujiwara et al., 1999). The reasons for this functional difference are not understood. Interestingly, a mutant of TthRRF lacking five residues from the Cterminal end complemented an E. coli strain defective in its $f r r$ gene (Fujiwara et al., 1999). The creation of similar mutations in E. coli RRF (EcoRRF) showed that mutants which lacked up to seven residues from the Cterminal end were still functional. However, deletions that resulted in the loss of nine or more residues from the C-terminal end were defective in ribosome recycling (Fujiwara et al., 2001; Janosi et al., 2000).
Recently, we have shown that, like TthRRF, M. tuberculosis RRF (MtuRRF) also failed to complement an $f r r^{\text {ts }}$ strain of E. coli LJ14. However, simultaneous expression of the elongation factor $G(E F G)$ and the RRF from M. tuberculosis resulted in the rescue of the temperature-sensitive phenotype of the LJ14 strain, highlighting the importance of specific interactions between the two proteins (Rao \& Varshney, 2001). To understand the mechanism of action of MtuRRF further, in this study we have generated a mutant of MtuRRF that lacks the last six amino acids of the C-terminal end (equivalent to $\Delta \mathrm{C} 5$ of $T$. thermophilus), investigated its biochemical and biophysical properties and carried out functional analyses in E. coli.

\section{METHODS}

E. coli strains and growth conditions. E. coli strains (Table 1) were grown in Luria-Bertani (LB) liquid or solid (with $1.5 \%$ agar) medium (Sambrook et al., 1989), unless stated otherwise. The medium was supplemented with tetracycline $\left(12.5 \mu \mathrm{g} \mathrm{ml}^{-1}\right)$ and ampicillin $\left(100 \mu \mathrm{g} \mathrm{ml}^{-1}\right)$ as required. When needed, IPTG was added to the medium to a final concentration of $0.5 \mathrm{mM}$. For the growth rate experiments, cultures were started with a $0.06 \%(\mathrm{v} / \mathrm{v})$ inoculum from freshly grown $\left(\right.$ at $30^{\circ} \mathrm{C}$ ) overnight cultures, and the growth at both the permissive $\left(30^{\circ} \mathrm{C}\right)$ and non-permissive $\left(42^{\circ} \mathrm{C}\right)$ temperatures was monitored by recording culture densities at $600 \mathrm{~nm}$ at regular intervals.

Cloning of RRF and EFG from $\boldsymbol{M}$. tuberculosis and $\boldsymbol{E}$. coli. The ORFs of the genes were cloned into vectors pTrc99C, pET11d and pACDH (Rao \& Varshney, 2001).

Construction of $\Delta \mathbf{C 6 M t u R R F}$. The mutant lacking six amino acids from the C-terminal end of $M t u R R F(\Delta C 6 M t u R R F)$ was generated by PCR from pTrcMtuRRF. PCR was carried out with $P f u$ DNA polymerase as described previously for $M t u R R F$ (Rao \& Varshney, 2001) using a forward primer $\left(5^{\prime}\right.$ GCGCCCATGGTTGATGAGGCTCTCTTC-3') containing an $\mathbf{N c o I}$ site and a reverse primer (5'-AGCAAAGCTTATTCTTTGTGTTTAACC-3') which incorporated a stop codon at position 180 ( $\mathrm{G}$ to amber) and a HindIII site. The 
Table 1. List of $E$. coli strains and plasmids used in this study

\begin{tabular}{|c|c|c|}
\hline Strain/plasmid & Relevant details* & Reference \\
\hline \multicolumn{3}{|l|}{ Strain } \\
\hline LJ14 & MC1061 containing frr $14^{\text {ts }}$ allele & Janosi et al. (1998) \\
\hline MRE600 & $\mathrm{F}^{-}, r n a$ & Gay (1984) \\
\hline BL21(DE3) & hsdS gal ( $\Delta$ cIts857 ind1 sam7 nin5 lacUV5-T7 gene 1$)$ & Studier \& Moffatt (1986) \\
\hline \multicolumn{3}{|l|}{ Plasmid } \\
\hline $\mathrm{pACDH}$ & $\begin{array}{l}\text { Harbours ACYC origin of replication; derived from pACD (Mangroo et al., } \\
\text { 1995) by mutating the HindIII site of the Tet }{ }^{\mathrm{R}} \text { marker to the NheI site }\end{array}$ & $\begin{array}{l}\text { T. K. DineshKumar \& U. } \\
\text { Varshney (unpublished } \\
\text { data) }\end{array}$ \\
\hline pACDHMtuRRF & M. tuberculosis RRF cloned into pACDH & Rao \& Varshney (2001) \\
\hline $\mathrm{pACDH} \Delta \mathrm{C} 6 \mathrm{M} t u \mathrm{RRF}$ & M. tuberculosis RRF ( $\triangle \mathrm{C} 6$ mutant) cloned into pACDH & This work \\
\hline pTrcEcoEFG & E. coli $\mathrm{EFG}$ cloned into pTrc99C & Rao \& Varshney (2001) \\
\hline pTrcMtuEFG & M. tuberculosis EFG cloned into pTrc99C & Rao \& Varshney (2001) \\
\hline pETMtuRRF & pET11d-based overexpression construct of $M t u$ EFG & Rao \& Varshney (2001) \\
\hline $\mathrm{pET} \Delta \mathrm{C} 6 \mathrm{M} t u \mathrm{RRF}$ & pET11d-based overexpression construct of $\Delta \mathrm{C} 6 \mathrm{M} t u \mathrm{RRF}$ & This work \\
\hline pETEcoRRF & pET11d-based overexpression construct of EcoRRF & Rao \& Varshney (2001) \\
\hline pETEcoEFG & pET11d-based overexpression construct of EcoEFG & Rao \& Varshney (2001) \\
\hline pETMtuEFG & pET11d-based overexpression construct of $M t u$ EFG & Rao \& Varshney (2001) \\
\hline
\end{tabular}

$* \mathrm{Tet}^{\mathrm{R}}$, tetracycline-resistant.

PCR product was digested with $\mathrm{NcoI}$ and HindIII and cloned into the respective sites of the ColE1 origin-of-replicationbased vectors pTrc99C and pET11d to generate $\mathrm{p} \operatorname{Trc} \Delta \mathrm{C} 6 M t u R R F$ and $\mathrm{pET} \Delta \mathrm{C} 6 \mathrm{M} t u \mathrm{RRF}$, respectively. The same fragment was also subcloned into pACDH containing a pACYC origin of replication to yield pACDH $\Delta$ C $6 M t u R R F$. Incorporation of the fragment was confirmed by complete DNA sequencing.

Purification of MtuRRF and $\Delta$ C6MtuRRF, N-terminal sequencing and electron spray ionization-mass spectroscopy (ESI-MS). $M t u$ RRF and $\Delta$ C6MtuRRF were purified from $E$. coli BL21(DE3) harbouring either pETMtuRRF or pET $\Delta$ C6MtuRRF, respectively. The transformants were inoculated into $2 \times \mathrm{YT}$ (Sambrook et al., 1989) (2 l) and induced with $0.5 \mathrm{mM}$ IPTG at the mid-exponential phase of growth $\left(\mathrm{OD}_{600}\right.$ value of between $0 \cdot 3$ and $\left.0 \cdot 4\right)$. Cells were harvested, sonicated and used to make the $\$ 100$ lysate. The S100 lysate was subjected to streptomycin sulfate $(0 \cdot 9 \%)$ precipitation, and the supernatant was subjected to ammonium sulfate precipitation ( $90 \%$ saturation). The precipitate was recovered by centrifugation, dissolved in $1 \mathrm{ml}$ of $20 \mathrm{mM}$ Tris/HCl $(\mathrm{pH} \mathrm{7.4)}$ and dialysed against the same buffer for $12 \mathrm{~h}$. Dialysed sample was loaded onto a Superdex 75 column (Amersham Pharmacia Biotech). Proteins were eluted with $20 \mathrm{mM}$ Tris/ $\mathrm{HCl}(\mathrm{pH} 7 \cdot 4), 500 \mathrm{mM} \mathrm{NaCl}$ and $10 \%(\mathrm{v} / \mathrm{v})$ glycerol, and the fractions enriched for RRF (as analysed by SDS-PAGE) were pooled, dialysed against $20 \mathrm{mM}$ Tris/ $\mathrm{HCl}$ ( $\mathrm{pH} 7 \cdot 4)$ and subjected to Mono Q column chromatography. The proteins were eluted with a linear gradient of $0-1 \mathrm{M} \mathrm{NaCl}$ in $20 \mathrm{mM}$ Tris/HCl $(\mathrm{pH} 7 \cdot 4)$ and $10 \%$ glycerol buffer. The fractions enriched for RRF were dialysed against $20 \mathrm{mM}$ HEPES (pH 5.0) and loaded onto a hydroxyapatite column (Bio-Rad); the RRF was eluted using $20 \mathrm{mM}$ HEPES (pH 5.0), $200 \mathrm{mM} \mathrm{NaCl}$ and $10 \%$ glycerol. The protein microsequence and ESI-MS analyses were carried out by the respective facilities at the Indian Institute of Science, Bangalore, India.

Purification of E. coli and M. tuberculosis EFG. EFGs were purified from E. coli BL21(DE3) using the T7 RNA polymerase expression constructs pETMtuEFG and pETEcoEFG (Rao \& Varshney, 2001).

Gel electrophoresis. Proteins were electrophoresed on 15 and $12 \%$ polyacrylamide gels containing $0 \cdot 1 \%$ SDS (SDS-PAGE) and visualized by Coomassie brillant blue R-250 staining (Laemmli, 1970). The non-denaturing PAGE (native PAGE) was performed in the same way but lacked SDS. Loading dye for native gels consisted of $50 \mathrm{mM}$ Tris $/ \mathrm{HCl}(\mathrm{pH} \mathrm{6.8)}, 10 \%$ $(\mathrm{v} / \mathrm{v})$ glycerol and $0.01 \%$ bromophenol blue.

Circular dichroism (CD) spectroscopy. CD measurements were done on an automated JASCO-J715 spectropolarimeter using $0 \cdot 2 \mathrm{~cm}$ and $1 \mathrm{~cm}$ path length quartz cuvettes for secondary and tertiary CD spectra, respectively. Samples were prepared in $20 \mathrm{mM}$ potassium phosphate buffer $(\mathrm{pH} \mathrm{7 \cdot 0)}$. Each spectrum was a mean of four scans with a slit width of $1 \mathrm{~nm}$, response time of $4 \mathrm{~s}$ and a scan speed of $50 \mathrm{~nm} \mathrm{~s}^{-1}$.

Polysome preparation, in vitro ribosome recycling and polysome binding assays. Polysomes were prepared from $E$. coli MRE600 and used in ribosome recycling assays (Tai \& Davis, 1979; Girbes et al., 1979; Rao \& Varshney, 2001). To perform binding assays, factor-free polysomes $\left(\mathrm{OD}_{260} \sim 2\right)$ were incubated in reaction volumes of $250 \mu \mathrm{l}$ without or with RRFs in the RRF assay buffer $(10 \mathrm{mM}$ Tris/ $\mathrm{HCl}, \mathrm{pH} 7 \cdot 4$, $80 \mathrm{mM} \mathrm{NH}_{4} \mathrm{Cl}, 8.2 \mathrm{mM} \mathrm{MgSO}{ }_{4}, 1 \mathrm{mM}$ DTT, $10 \mu \mathrm{M}$ puromycin, $160 \mu \mathrm{M} \mathrm{GTP}$ ) at $33^{\circ} \mathrm{C}$ for $20 \mathrm{~min}$ and the reaction mixture was layered on a mini-column $(1 \mathrm{ml})$ packed with Sepharose 4B matrix and centrifuged at 2500 r.p.m. for 3 min in a table-top centrifuge. The eluate was concentrated by vacuum drying, separated by SDS-PAGE $(15 \%)$ and analysed by immunoblotting (Towbin et al., 1979).

Immunoblotting. The cell-free extracts $(10 \mu \mathrm{g}$ total proteins) or the ribosome samples (in the RRF binding studies) were separated by SDS-PAGE $(12 \%)$ and electroblotted onto a PVDF membrane (Towbin et al., 1979) at $200 \mathrm{~mA}$ for $2 \mathrm{~h}$. The blots were probed with anti-MtuRRF rabbit antibodies and detected using alkaline phosphatase-conjugated goat-antirabbit IgG with the substrates $p$-nitrotetrazolium blue chloride and 5-bromo-4-chloro-3-indolyl phosphate. 


\section{RESULTS}

\section{Purification of RRF}

MtuRRF ( $\sim 21 \mathrm{kDa})$ was purified to apparent homogeneity by chromatography on Superdex 75 , MonoQ and hydroxyapatite columns (Fig. 2a). Microsequencing of this preparation yielded an $\mathrm{N}$-terminal sequence,
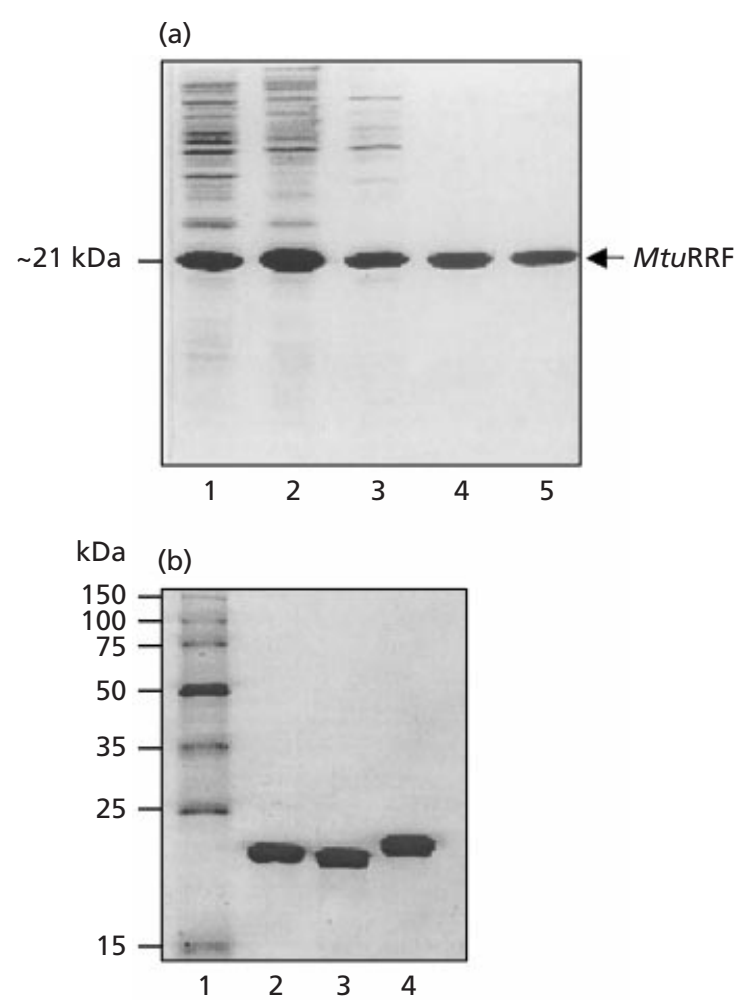

(c)

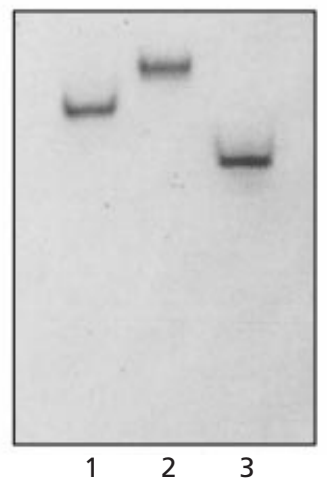

Fig. 2. Purification and characterization of RRFs. (a) Purification profile of MtuRRF on SDS-PAGE (15\%). Lanes: 1 and 2, aliquots $(\sim 8 \mu \mathrm{g})$ from S20 and S100 extracts, respectively; 3-5, aliquot $(\sim 4 \mu \mathrm{g})$ from the pool of eluates from Superdex 75 , Mono Q and hydroxyapatite columns, respectively. (b) Analysis of purified RRFs ( $\sim 4 \mu \mathrm{g}$ each protein) on SDS-PAGE (15\%). Lanes: 1, molecular mass marker (Sigma); 2, $\Delta$ C6MtuRRF; 3, EcoRRF; 4, MtuRRF. (c) Analysis of purified RRFs on $15 \%$ PAGE (native). Lanes: 1, $\Delta$ C6MtuRRF $(\sim 2 \mu \mathrm{g}) ; 2$, ECoRRF $(\sim 2 \mu \mathrm{g}) ; 3$, MtuRRF $(\sim 3 \mu \mathrm{g})$.
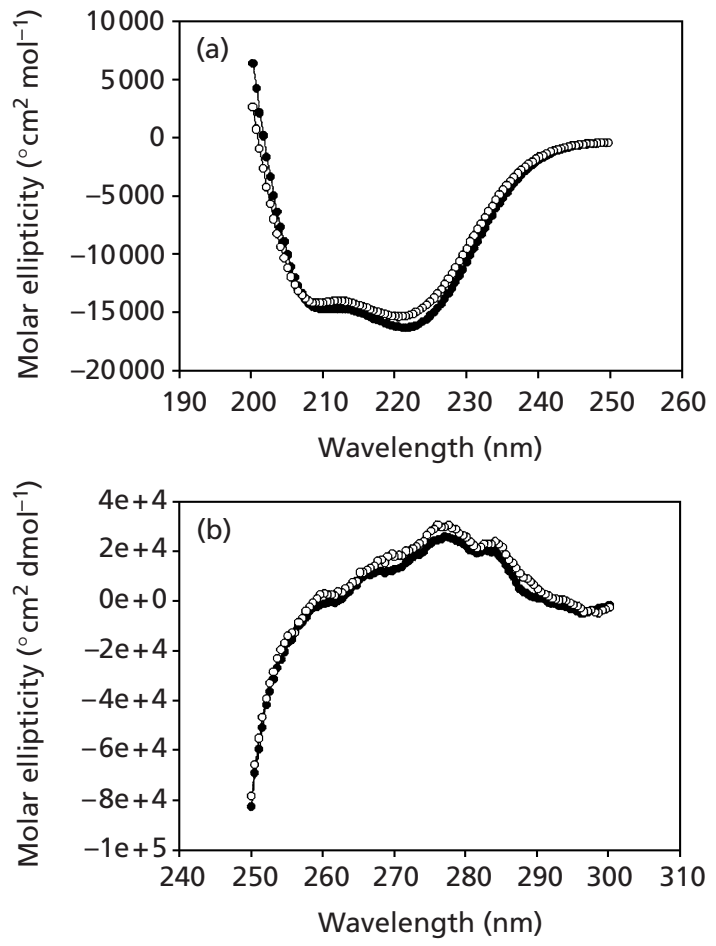

Fig. 3. $C D$ spectroscopy. (a) Secondary structure and (b) tertiary structure CD spectra of MtuRRF $(0, a ; O, b)$ and $\triangle C 6 M t u R R F$ $(\bigcirc, a ; 0, b)$.

\begin{tabular}{|c|c|c|c|c|c|c|c|c|c|c|}
\hline Mtu RRF $(\mu \mathrm{g})$ & + & - & - & 5 & 5 & 5 & 5 & - & 5 & - \\
\hline$\triangle \mathrm{C} 6 \mathrm{MtuRRF}(\mu \mathrm{g})$ & - & 5 & 50 & 5 & 25 & 50 & - & - & 50 & + \\
\hline $\begin{array}{r}\text { MtuRRF } \\
\Delta C 6 M t u R R F\end{array}$ & $=$ & 4 & & & $=$ & $\pi$ & $\min$ & 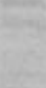 & & $\infty$ \\
\hline & M1 & 1 & 2 & 3 & 4 & 5 & 6 & 7 & 8 & M2 \\
\hline
\end{tabular}

Fig. 4. Binding of MtuRRF and $\Delta C 6 M t u R R F$ to $E$. coli polysomes. The binding to polysomes was analysed using a spin-column method (see Methods). Lanes: 1, $\Delta$ C6MtuRRF alone $(5 \mu \mathrm{g}) ; 2$, $\Delta$ C6MtuRRF alone $(50 \mu \mathrm{g}) ; 3$, MtuRRF $(5 \mu \mathrm{g})$ and $\Delta$ C6MtuRRF $(5 \mu \mathrm{g}) ; 4, M$ tuRRF $(5 \mu \mathrm{g})$ and $\Delta$ C6MtuRRF $(25 \mu \mathrm{g}) ; 5, M t u R R F$ $(5 \mu \mathrm{g})$ and $\Delta$ C6MtuRRF $(50 \mu \mathrm{g}) ; 6, \operatorname{MtuRRF}(5 \mu \mathrm{g}) ; 7$, polysomes alone; 8, MtuRRF $(5 \mu \mathrm{g})$ and $\Delta$ C6MtuRRF $(50 \mu \mathrm{g})$ without polysomes; $\mathrm{M} 1$ and $\mathrm{M} 2$, marker lanes for MtuRRF and $\triangle$ C6MtuRRF, respectively.

?DEALFDAEE ..., which matched with the N-terminal of MtuRRF sequence deduced from its gene, suggesting that the initiator $\mathrm{f}^{\mathrm{Met}}$ was cleaved from the mature protein. The ESI-MS analysis also showed a major peak of $20720 \mathrm{Da}$ that corresponded to the protein lacking the $\mathrm{N}$-terminal $\mathrm{f}^{\mathrm{Met}}$. However, in this analysis, a minor peak of $20851 \mathrm{Da}$ corresponding to a polypeptide in which $\mathrm{f}^{\mathrm{Met}}$ had not been removed was also seen. This was most likely due to hyperexpression of MtuRRF. 
(a)
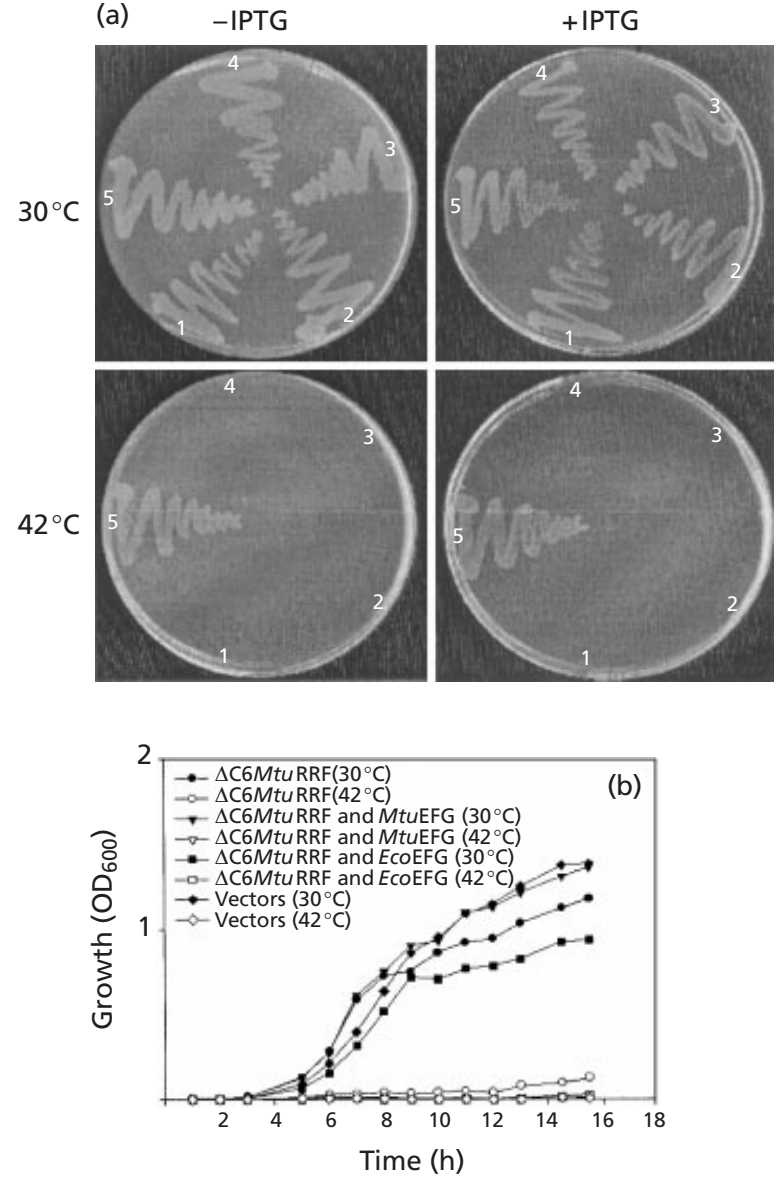

(c)

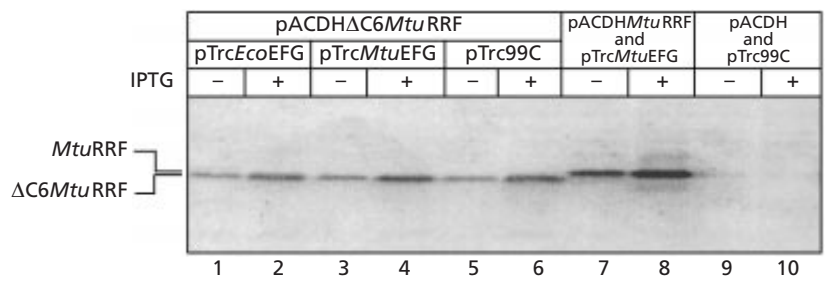

Fig. 5. Analysis of complementation of E. coli LJ14 ( $\left.\mathrm{frr}^{\mathrm{ts}}\right)$ with $\Delta$ C6MtuRRF. (a) Various plasmids were introduced into $E$. coli LJ14 and the fresh cultures of the transformants (uninduced or induced with $0.5 \mathrm{mM}$ IPTG) were streaked onto LB agar plates containing ampicillin and tetracycline with or without IPTG, respectively, in duplicate. One plate was incubated at the permissive temperature $\left(30^{\circ} \mathrm{C}\right)$ and the other at the nonpermissive temperature $\left(42^{\circ} \mathrm{C}\right)$. Sectors: $1, \mathrm{pACDH}$ and pTrc $99 \mathrm{C}$; 2, pACDH $\Delta$ C6MtuRRF and pTrc99C; 3, pACDH $\Delta C 6 M t u R R F$ and pTrCMtuEFG; 4, pACDH $\triangle$ C6MtuRRF and pTrCECOEFG; 5, pACDHMtuRRF and pTrcMtuEFG. (b) Growth of E. coli LJ14 $\left(f r r^{t s}\right)$ transformants at the permissive $\left(30^{\circ} \mathrm{C}\right)$ and nonpermissive $\left(42{ }^{\circ} \mathrm{C}\right)$ temperatures. Cultures in LB containing ampicillin, tetracycline and $0.5 \mathrm{mM}$ IPTG were started with $0.06 \%$ inoculums from overnight cultures $\left(30^{\circ} \mathrm{C}\right)$ and were grown at the permissive $\left(30^{\circ} \mathrm{C}\right.$, solid symbols) and nonpermissive $\left(42{ }^{\circ} \mathrm{C}\right.$, open symbols) temperatures. The growth of the cultures $\left(O D_{600}\right)$ was monitored at regular intervals. The transformants harboured either vectors alone ( $\mathrm{pACDH}$ and pTrc99C) or various recombinants expressing RRF and EFG, as indicated in the inset. (c) Detection of $\Delta$ C6MtuRRF expression in E. coli LJ14 by immunoblotting using anti-MtuRRF antibodies. Protein extracts $(10 \mu \mathrm{g}$ total protein) prepared from cultures
$\Delta \mathrm{C} 6 \mathrm{M} t u \mathrm{RRF}$ and EcoRRF were also purified to apparent homogeneity using the methodology described above. The comparative analysis of the full-length RRFs (MtuRRF and EcoRRF) by SDS-PAGE showed that in spite of their similar sizes (185 aa each) and closely related molecular masses, MtuRRF (Fig. 2b, lane 4) migrated somewhat slower than EcoRRF (Fig. 2b, lane 3). As expected, $\Delta \mathrm{C} 6 M t u R R F$ migrated slightly faster than the wild-type form, MtuRRF (Fig. 2b, compare lanes 2 and 4). However, the migration of the three RRFs on native PAGE (Fig. 2c) was according to their calculated pI values $(6 \cdot 16,6.45$ and 5.71 for $\Delta \mathrm{C} 6 \mathrm{M} t u \mathrm{RRF}, E c o \mathrm{RRF}$ and MtuRRF, respectively). Since all three of the RRFs had similar molecular masses $(20 \cdot 18,20 \cdot 84$ and $20 \cdot 82 \mathrm{kDa}$, respectively), the observed mobility profile on the native gel implied that the overall architecture of these proteins was likely to be similar.

\section{CD spectroscopy}

The X-ray structures of RRFs have shown that the two domains that mimic the arms of the ' $L$ '-shaped tRNA consist of well-defined secondary structural elements (Yoshida et al., 2001; Selmer et al., 1999; Kim et al., 2000; Toyada et al., 2000). Domain I consists of three long $\alpha$-helices. This property of RRF makes it a suitable molecule for CD spectroscopic analysis to probe for any major structural changes that could result from the introduction of mutations. As seen in Fig. 3(a), the secondary CD profiles of the wild-type (MtuRRF) and the mutant $(\Delta \mathrm{C} 6 \mathrm{M} t u \mathrm{RRF})$ proteins are almost identical, suggesting that the deletion of six residues from the Cterminal end of MtuRRF does not result in its inappropriate folding. Furthermore, although the tertiary CD signals are weak (MtuRRF lacks tryptophans), the similar spectra for the two proteins (Fig. $3 \mathrm{~b}$ ) suggest that even the overall architecture of the two proteins is similar.

\section{Polysome binding assays}

We carried out polysome binding assays by a novel spincolumn method using Sepharose 4B matrix, which permitted elution of ribosome-bound RRF but not free RRF into the excluded volume (Fig. 4). This analysis showed that although $\Delta \mathrm{C} 6 \mathrm{M} t u \mathrm{RRF}$ and MtuRRF bound to ribosomes (Fig. 4, lanes 1 and 6, respectively), the binding of $\Delta \mathrm{C} 6 \mathrm{MtuRRF}$ was weaker than that of $M t u R R F$. However, when $\Delta \mathrm{C} 6 M t u R R F$ was taken in a large amount $(50 \mu \mathrm{g})$, its binding to ribosomes was comparable to that of the full-length protein $(5 \mu \mathrm{g})$ (Fig. 4, compare lanes 2 and 6). And, as expected, when the free polysomes, or the same amounts of free RRFs, were spun through the mini-columns, no signals corresponding to RRFs were seen (Fig. 4, lanes 7 and 8, respectively).

induced $(+)$ with $0.5 \mathrm{mM}$ IPTG or uninduced $(-)$ cultures were separated by SDS-PAGE, transferred to a PVDF membrane and then probed with anti-MtuRRF antibodies. Details of the various plasmids in the transformants are as shown. 
(a)

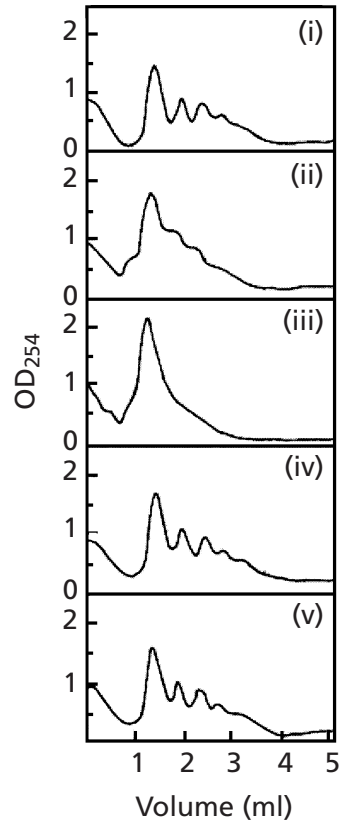

(b)

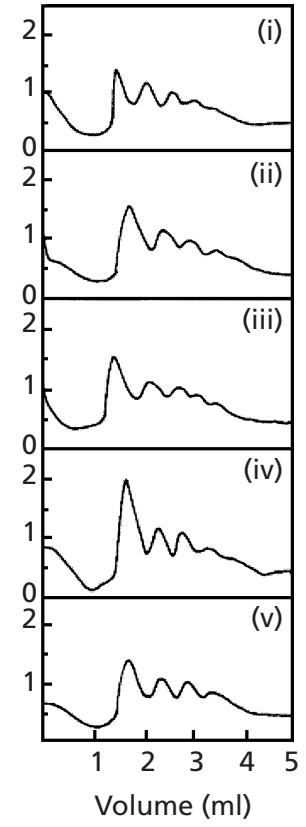

(c)

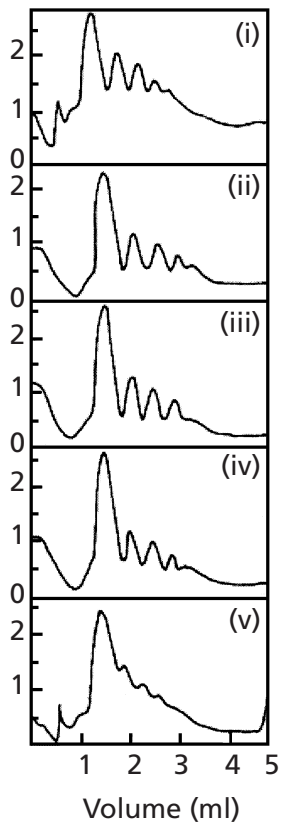

Fig. 6. RRF activity assays using $E$. coli polysomes. (a) Assays with MtuRRF and MtuEFG. (i) Polysomes alone, (ii) MtuRRF $(10 \mu \mathrm{g})$ and MtuEFG $(20 \mu \mathrm{g})$, (iii) MtuRRF $(20 \mu \mathrm{g})$ and MtuEFG $(40 \mu \mathrm{g})$, (iv) MtuRRF $(20 \mu \mathrm{g})$, and (v) MtuEFG $(40 \mu \mathrm{g})$. (b) Assays with $\triangle$ C6MtuRRF and MtuEFG. (i) Polysomes alone, (ii) $\Delta$ C6MtuRRF $(20 \mu \mathrm{g})$ and MtuEFG $(40 \mu \mathrm{g})$, (iii) $\Delta$ C6MtuRRF $(40 \mu \mathrm{g})$ and MtuEFG $(80 \mu \mathrm{g})$, (iv) $\Delta$ C6MtuRRF $(40 \mu \mathrm{g})$, and (v) MtuEFG $(80 \mu \mathrm{g}) . \quad$ (c) Assays with mycobacterial and $E$. coli proteins. (i) Polysomes alone, (ii) $\Delta$ C6MtuRRF $(1 \mu \mathrm{g})$ and ECoEFG $(4 \mu \mathrm{g})$, (iii) $\Delta$ C6MtuRRF $(4 \mu \mathrm{g})$ and ECoEFG $(16 \mu \mathrm{g})$, (iv) $\Delta$ C6MtuRRF $(8 \mu \mathrm{g})$ and ECoEFG $(32 \mu \mathrm{g})$, and (v) ECoRRF $(2 \mu \mathrm{g})$ and ECoEFG $(15 \mu \mathrm{g})$.
Furthermore, we carried out competitive binding analyses of the two RRFs to polysomes (Fig. 4, lanes 3-5). As seen from the band intensities corresponding to the two proteins, in the presence of a fixed amount of MtuRRF $(5 \mu \mathrm{g})$ and increasing amounts of $\Delta \mathrm{C} 6 \mathrm{MtuRRF}$ (5 to $50 \mu \mathrm{g}$ ), the binding of the latter to ribosomes increased with a concomitant decrease in the binding of MtuRRF. Consistent with the analyses in Figs $2(b)$ and $3(a, b)$, the ability of $\Delta \mathrm{C} 6 \mathrm{M} t u \mathrm{RRF}$ to bind to the ribosomes further supported the view that it folded correctly.

\section{Complementation analysis of E. coli LJ14 with IC6MtuRRF}

As the deletion of the C-terminal five amino acids from TthRRF bestowed upon it the ability to complement an $\mathrm{frr}^{\text {ts }}$ strain of E. coli, it was of interest to us to investigate the phenotype of an equivalent mutant of MtuRRF. However, our analysis showed that $\Delta \mathrm{C} 6 \mathrm{M} t u \mathrm{RRF}$ failed to rescue the temperature-sensitive phenotype of E. coli LJ14 $\left(f r r^{\mathrm{ts}}\right)$ even upon its induction with IPTG (Fig. 5a, sector 2, compare growth at the permissive temperature to growth at the non-permissive temperature). Simultaneous overproduction of E. coli EFG (EcoEFG) also did not confer the ability to $\Delta \mathrm{C} 6 \mathrm{MtuRRF}$ to rescue the temperature-sensitive phenotype of E. coli LJ14 at the non-permissive temperature (Fig. 5a, sector 3). In fact, $\Delta$ C6MtuRRF failed to complement E. coli LJ14 even when co-expressed with $M$. tuberculosis EFG (MtuEFG) (Fig. 5a, sector 4). However, as reported earlier (Rao \& Varshney, 2001), the wild-type MtuRRF complemented E. coli LJ14 in the presence of MtuEFG (Fig. 5a, sector 5) irrespective of induction with IPTG. To further verify these results, we monitored the growth of the transformants in liquid cultures (Fig. 5b). Consistent with the plating experiment, the transformants harbouring the vectors or the $\Delta \mathrm{C} 6 \mathrm{MtuRRF}$ constructs alone or along with the plasmids harbouring EcoEFG or MtuEFG grew at the permissive temperature but not the non-permissive temperature. The immunoblot analysis of the cellular extracts of the transformants (grown at the permissive temperature) showed that $\Delta \mathrm{C} 6 \mathrm{M} t u \mathrm{RRF}$ was produced in E. coli LJ14 (Fig. 5c, lanes 1-6) and its expression increased upon induction with IPTG (Fig. 5c, compare lanes 1,3 and 5 with 2, 4 and 6). The levels of $\Delta$ C6MtuRRF produced upon induction with IPTG (Fig. 5 c, lanes 2, 4 and 6) were comparable to those of wildtype MtuRRF in uninduced cultures (Fig. 5c, lane 7), suggesting that the failure of complementation of $E$. coli LJ14 by the mutant protein was not due to its inadequate production.

\section{In vitro activity of $\triangle \mathrm{C} 6 M$ tuRRF with homologous EFG (MtuEFG) and heterologous EFG (EcoEFG)}

To rule out the possibility that the failure of complementation of E. coli LJ14 by $\Delta \mathrm{C} 6 M t u$ RRF was due to the temperature-sensitive nature of the mutant per se, we carried out in vitro polysome disassembly assays. Fig. 6 shows that the polysome preparation afforded a distinct profile consisting of the monosome and multimer $(2 \times$, $3 \times, 4 \times, 5 \times$, etc.) peaks on the sucrose gradients (Fig. 6 , panel i). When the polysomes were treated with either $M t u$ RRF or $M t u$ EFG no discernible changes in the profiles were observed (Fig. 6a, compare iv and v to i). As expected, when the polysomes were treated with the two proteins together (Fig. 6a, ii and iii), commensurate with their amounts, the polysomes were converted into the monomers. However, $\Delta \mathrm{C} 6 \mathrm{MtuRRF}$ neither alone (Fig. 6b, iv) nor with EcoEFG (Fig. 6c, ii-iv) resulted in any detectable polysome breakdown. More importantly, and consistent with the in vivo analysis, $\Delta \mathrm{C} 6 \mathrm{MtuRRF}$ 
failed to convert the polysomes to monomers even in the presence of homologous EFG (MtuEFG) in spite of the presence of high concentrations of the proteins (Fig. 6b; ii, $20 \mu \mathrm{g}$ of $\Delta \mathrm{C} 6 \mathrm{MtuRRF}$ with $40 \mu \mathrm{g}$ of MtuEFG; iii, $40 \mu \mathrm{g}$ of $\Delta \mathrm{C} 6 \mathrm{MtuRRF}$ with $80 \mu \mathrm{g}$ of $\mathrm{MtuEFG).} \mathrm{In} \mathrm{yet}$ another control experiment, we carried out polysome disassembly assays using EcoRRF to ensure that EcoEFG was active. As seen in Fig. 6(c), EcoRRF converted the polysomes into monosomes in the presence of EcoEFG (Fig. 6c, v). These in vitro assays support the in vivo analysis shown in Fig. 5.

\section{DISCUSSION}

Termination of protein synthesis releases the nascent polypeptide chains from the ribosomes (Hershey, 1987). However, the mechanistic events that lead to the disassembly of the post-termination complexes and recycling of ribosomes for a fresh round of protein synthesis are still being unravelled. Previous biochemical and genetic analyses have established a key role for RRF in disassembly of the termination complex (Janosi et al., 1996, 2000; Karimi et al., 1999). RRF is unique to prokaryotic protein synthesis, and as demonstrated using E. coli, it is essential for the survival of bacteria (Janosi et al., 1994; Inokuchi et al., 2000). However, the gaps in our understanding of the mechanistic aspects of RRF function present a major limitation in the exploitation of this factor as a novel drug target. Mutational analyses, a crucial facet of structure-function studies, are important for a better understanding of the mechanism of action of RRF (Janosi et al., 2000; Fujiwara et al., 2001).

It has been shown that RRF from $P$. aeruginosa complements E. coli LJ14 ( $\left.f r r^{\mathrm{ts}}\right)$ (Ohnishi et al., 1999); however, TthRRF does not complement this mutant strain. Interestingly, a mutant lacking five amino acids from the C-terminal end of TthRRF did complement the $\mathrm{frr}^{\mathrm{ts}}$ phenotype of E. coli (Fujiwara et al., 1999; Toyoda et al., 2000). Furthermore, recent studies using EcoRRF have shown that deletion of up to seven amino acids from the C-terminal end of this protein still allows it to retain its activity (Fujiwara et al., 2001). Therefore, these C-terminal residues are not absolutely critical for RRF function. Alignment of the available RRF sequences (a total of 49 ) showed that amino acid residues 178-185 (E. coli numbering) belong to a highly conserved stretch of amino acids in RRFs. Does this high degree of conservation allude to the importance of these residues in RRF function?

In this study, based on the sequence comparison of $M t u R R F$ and TthRRF (Fig. 1), we generated a mutant of $M t u R R F$ that lacked the last six amino acids from the Cterminal end $(\Delta \mathrm{C} 6 \mathrm{MtuRRF})$. However, unlike the Cterminally deleted TthRRF or EcoRRF mutants, $\Delta$ C6MtuRRF failed to function with EcoEFG in ribosome recycling in both in vivo and in vitro analyses. Surprisingly, $\Delta \mathrm{C} 6 \mathrm{M} t u \mathrm{RRF}$ even failed to function with MtuEFG (Figs 5 and 6). The biochemical and biophysical characterizations performed here suggest that the mutant protein is folded properly and retains a shape similar to that of the wild-type protein. Interestingly, the ribosome binding assays (Fig. 4) show that while $M t u$ RRF binds to E. coli ribosomes, the mutant protein is compromised for its binding to ribosomes. And, while such binding studies have not been carried out with the equivalent mutants of TthRRF and EcoRRF, we suggest that the loss of ribosome binding activity of the $\Delta \mathrm{C} 9$ mutants of EcoRRF (Fujiwara et al., 2001; Toyoda et al., 2000) is predominantly a consequence of the loss of these highly conserved residues at the C-terminal end of the RRF. Thus, the conserved residues at the C-terminal end of the RRFs may facilitate in their direct binding to ribosomes and/or in their prolonged residency on the ribosomes, possibly by modulating the 'on' and/or 'off' rates.

T. thermophilus and E. coli belong to the Gram-negative group of bacteria, whereas $M$. tuberculosis belongs to the Gram-positive group, indicating that TthRRF is relatively closer to EcoRRF and, therefore, may already possess many of the elements that are needed to establish specific contacts with EFG and the ribosome. Alignment of the RRF sequences (Fig. 1) shows that out of 185 residues a total of 74 are conserved between MtuRRF and EcoRRF ( $\sim 40 \%$ sequence identity) with a similarity score of $\sim 59 \%(109 / 185)$. However, a total of 81 residues are conserved between TthRRF and EcoRRF ( $\sim 44 \%$ sequence identity) with a similarity score of $\sim 63 \%(116 / 185)$. Thus, there is slightly higher sequence similarity between EcoRRF and TthRRF than between EcoRRF and MtuRRF. It has been suggested that a deletion of five amino acids from the C-terminal end of TthRRF results in improved flexibility of the hinge region connecting domains I and II of RRF, which in turn may facilitate its function in E. coli (Toyoda et al., 2000). Possibly, what the removal of the residues from the C-terminal end achieves is that it brings these interacting partners into a better configuration for a productive association, such that even the shorter residency times of $T t h R R F$ are now adequate. However, in MtuRRF, which in comparison to TthRRF is slightly less intimately related to EcoRRF, an equivalent deletion may not result in similar favourable changes. It should also be noted that the earlier study (Fujiwara et al., 1999) utilized a temperature-sensitive strain of E. coli containing a different allele of $f r r$ than the one used in this study. Thus, at this stage alternative interpretations that relate to the strain effects can not be ruled out. Nevertheless, our studies do highlight the significance of RRF binding to ribosomes and the establishment of specific interactions between RRF and EFG for the disassembly of post-termination complexes.

\section{ACKNOWLEDGEMENTS}

We thank Dr A. Kaji, University of Pennsylvania, USA, for kindly providing us with E. coli LJ14. We also thank Sarathkumar Kalapala, Shaillay Dogra, N. Acharya and other colleagues for their invaluable help in constructing several of the figures and for their useful suggestions. The support rendered by the protein microsequencing and ESI-MS facilities 
is gratefully acknowledged. This work was supported by research grants from the Department of Biotechnology and from the Department of Science and Technology, Government of India, New Delhi.

\section{REFERENCES}

Clark-Curtis, J. E. (1990). Genome structure of mycobacteria. In Molecular Biology of the Mycobacteria, pp. 77-95. Edited by J. McFadden. London: Academic Press.

Cole, S. T., Brosch, R., Parkhill, J. \& 39 other authors. (1998). Deciphering the biology of Mycobacterium tuberculosis from the complete genome sequence. Nature 393, 537-544.

Fujiwara, T., Ito, K., Nakayashiki, T. \& Nakamura, Y. (1999). Amber mutations in ribosome recycling factors of Escherichia coli and Thermus thermophilus: evidence for C-terminal modulator element. FEBS Lett 447, 297-302.

Fujiwara, T., Ito, K. \& Nakamura, Y. (2001). Functional mapping of ribosome-contact sites in the ribosome recycling factor: a structural view from a tRNA mimic. RNA 7, 64-70.

Gay, N. J. (1984). Construction and characterization of an Escherichia coli strain with a uncl mutation. J Bacteriol 158, 820-825.

Girbes, T., Cabrer, B. \& Modolell, J. (1979). Preparation and assay of purified Escherichia coli polysomes devoid of free ribosomal subunits and endogenous GTPase activities. Methods Enzymol 59, 353-362.

Hershey, J. W. B. (1987). In Escherichia coli and Salmonella typhimurium: Cellular and Molecular Biology, pp. 613-647. Edited by F. Neidhardt and others. Washington, DC: American Society for Microbiology.

Inokuchi, Y., Hirashima, A., Sekine, Y., Janosi, L. \& Kaji, A. (2000). Role of ribosome recycling factor (RRF) in translational coupling. EMBO J 19, 3788-3798.

Janosi, L., Shimizu, I. \& Kaji, A. (1994). Ribosome recycling factor (ribosome releasing factor) is essential for bacterial growth. Proc Natl Acad Sci U S A 91, 4249-4253.

Janosi, L., Hara, H., Zhang, S. \& Kaji, A. (1996). Ribosome recycling by ribosome recycling factor (RRF) - an important but overlooked step of protein biosynthesis. Adv Biophys 32, 121-201.

Janosi, L., Mottagui-Tabar, S., Isaksson, L. A. \& 7 other authors (1998). Evidence for in vivo ribosome recycling, the fourth step in protein biosynthesis. EMBO J 17, 1141-1151.

Janosi, L., Mori, H., Sekine, Y., Abragan, J., Janosi, R., Hirokawa, G. \& Kaji, A. (2000). Mutations influencing the $f r r$ gene coding for ribosome recycling factor (RRF). J Mol Biol 295, 815-829.

Kaji, A., Teyssier, E. \& Hirokawa, G. (1998). Disassembly of the post-termination complex and reduction of translational error by ribosome recycling factor $(\mathrm{RRF})-\mathrm{a}$ possible new target for antibacterial agents. Biochem Biophys Res Commun 250, 1-4.

Karimi, R., Pavlov, M. Y., Buckingham, R. H. \& Ehrenberg, M. (1999). Novel roles for classical factors at the interface between translation termination and initiation. Mol Cell 3, 601-609.

Kaufmann, S. H. \& van Embden, J. D. (1993). Tuberculosis: a neglected disease strikes back. Trends Microbiol 1, 2-5.
Kim, K. K., Min, K. \& Suh, S. W. (2000). Crystal structure of the ribosome recycling factor from Escherichia coli. EMBO J 19, 2362-2370.

Laemmli, U. K. (1970). Cleavage of structural proteins during the assembly of the head of bacteriophage T4. Nature 227, 680-685.

Lowe, A. M., Beattie, D. T. \& Deresiewicz, R. L. (1998). Identification of novel staphylococcal virulence genes by in vivo expression technology. Mol Microbiol 27, 967-976.

Mangroo, D., Limbach, P. A., McCloskey, J. A. \& RajBhandary, U. L. (1995). An anticodon sequence mutant of Escherichia coli initiator tRNA: possible importance of a newly acquired base modification next to the anticodon on its activity in initiation. J Bacteriol 177, 2858-2862.

McFadden, J. (1996). Recombination in mycobacteria. Mol Microbiol 21, 205-211.

Ohnishi, M., Janosi, L., Shuda, M., Matsumoto, H., Hayashi, T., Terawaki, Y. \& Kaji, A. (1999). Molecular cloning, sequencing, purification, and characterization of Pseudomonas aeruginosa ribosome recycling factor. J Bacteriol 181, 1281-1291.

Rao, A. R. \& Varshney, U. (2001). Specific interaction between the ribosome recycling factor and the elongation factor $G$ from Mycobacterium tuberculosis mediates peptidyl-tRNA release and ribosome recycling in Escherichia coli. EMBO J 20, 2977-2986.

Sambrook, J., Fritsch, E. F. \& Maniatis, T. (1989). Molecular Cloning: a Laboratory Manual, 2nd edn. Cold Spring Harbor, NY: Cold Spring Harbor Laboratory.

Selmer, M., Al-Karadaghi, S., Hirokawa, G., Kaji, A. \& Liljas, A. (1999). Crystal structure of Thermotoga maritima ribosome recycling factor: a tRNA mimic. Science 286, 2349-2352.

Studier, F. W. \& Moffatt, B. A. (1986). Use of bacteriophage T7 RNA polymerase to direct selective high-level expression of cloned genes. J Mol Biol 189, 113-130.

Tai, P. C. \& Davis, B. D. (1979). Isolation of polysomes free of initiation factors. Methods Enzymol 59, 362-371.

Towbin, H., Staehelin, T. \& Gordon, J. (1979). Electrophoretic transfer of proteins from polyacrylamide gels to nitrocellulose sheets: procedure and some applications. Proc Natl Acad Sci US A 76, 4350-4354.

Toyoda, T., Tin, O. F., Ito, K., Fujiwara, T., Kumasaka, T., Yamamoto, M., Garber, M. B. \& Nakamura, Y. (2000). Crystal structure combined with genetic analysis of the Thermus thermophilus ribosome recycling factor shows that a flexible hinge may act as a functional switch. RNA 6, 1432-1444.

Vizcaino, N., Cloeckaert, A., Dubray, G. \& Zygmunt, M. S. (1996). Cloning, nucleotide sequence, and expression of the gene coding for a ribosome recycling factor-homologous protein of Brucella melitensis. Infect Immun 64, 4834-4837.

Yoshida, T., Uchiyama, S., Nakano, H. \& 11 other authors (2001). Solution structure of the ribosome recycling factor from Aquifex aeolicus. Biochemistry 40, 2387-2396.

Young, D. B. \& Cole, S. T. (1993). Leprosy, tuberculosis, and the new genetics. J Bacteriol 175, 1-6.

Received 2 April 2002; revised 1 July 2002; accepted 29 August 2002. 